\title{
An SEIRS epidemic model with stochastic transmission
}

Peter J Witbooi*

\section{"Correspondence:}

pwitbooi@uwc.ac.za

Department of Mathematics and Applied Mathematics, University of the Western Cape, Robert Sobukwe Rd, Bellville, 7530, South Africa

\section{Springer}

\begin{abstract}
For an SEIRS epidemic model with stochastic perturbations on transmission from the susceptible class to the latent and infectious classes, we prove the existence of global positive solutions. For sufficiently small values of the perturbation parameter, we prove the almost surely exponential stability of the disease-free equilibrium whenever a certain invariant $\mathcal{R}_{\sigma}$ is below unity. Here $\mathcal{R}_{\sigma}<\mathcal{R}$, the latter being the basic reproduction number of the underlying deterministic model. Biologically, the main result has the following significance for a disease model that has an incubation phase of the pathogen: A small stochastic perturbation on the transmission rate from susceptible to infectious via the latent phase will enhance the stability of the disease-free state if both components of the perturbation are non-trivial; otherwise the stability will not be disturbed. Simulations illustrate the main stability theorem.
\end{abstract}

Keywords: SEIRS model; stochastic transmission; almost sure exponential stability

\section{Introduction}

In recent years, a number of articles have been published on stochastic differential equation models of population dynamics of infectious diseases. In comparison with models described by ordinary differential equations (ode), the stochastic differential equation (sde) models provide of course a means of accommodating randomness in the model. Two themes of special interest in the modeling of population dynamics of a disease are the stability of equilibrium points and the optimal control of interventions such as vaccination, quarantine, public health education and others. For sde models, optimal control problems and solutions are presented in [1] of Cai and Luo, [2] of Ishikawa and in [3]. In the stochastic setting, stability of equilibria and the long term persistence or extinction of a disease in a population have been studied in most of the sde models in the literature. Such studies use different versions of stability. Stochastic perturbation has also been studied in multigroup models, such as in [4-6] for example. In many cases it has been proved that the introduction of stochastic perturbations into an ode epidemic model system can possibly render an unstable disease-free equilibrium of the ode system to become stable in the stochastic differential equation system. This phenomenon was highlighted in [7] by Chen et al., [8] by Gray et al. and in [9] for instance.

Since the basic models such as [10] by Li et al. on diseases of the SEIRS type, many variations have been presented in the literature, such as [11] of Melesse and Gumel. Starting with an ode model of SEIRS type, in this paper we study the effect of stochastic pertur-

(c) The Author(s) 2017. This article is distributed under the terms of the Creative Commons Attribution 4.0 International License (http://creativecommons.org/licenses/by/4.0/), which permits unrestricted use, distribution, and reproduction in any medium, provided you give appropriate credit to the original author(s) and the source, provide a link to the Creative Commons license, and indicate if changes were made. 
bations on the stability of the disease-free equilibrium of the system. The models in [7] and in [8] have perturbations of the transmission rate from the $S$-class to the $I$-class. The latter models do not include a latent infection compartment such as the $E$ compartment in SEIR type models. The current paper is among the first studies of a disease model with a latent infection compartment, having a perturbation of the disease transmission. In the literature there are stochastic models such as in [4] and [5] having latent infection compartments, but with the stochastic perturbations not directly aimed at transmission. We prove the existence of solutions which are almost surely global and positive. We also study stability of the disease-free equilibrium. In particular, we introduce an invariant $\mathcal{R}_{\sigma}$ of the model that is related to the basic reproduction number $\mathcal{R}$ of the underlying deterministic model, with $\mathcal{R}_{\sigma}<\mathcal{R}$. With the given type of randomness in the system, we prove that there is a greater chance of the disease vanishing from the population. The main results are illustrated with simulations.

\section{Preliminaries}

Notation 2.1 By $\mathbb{R}_{+}^{n}$ (resp. $\mathbb{R}_{++}^{n}$ ) we denote the set of points in $\mathbb{R}^{n}$ having only non-negative (resp. strictly positive) coordinates.

We assume throughout the paper that we have a complete probability space $(\Omega, \mathcal{F}, \mathbb{P})$, equipped with a filtration, $\left\{\mathcal{F}_{t}\right\}_{t \geq 0}$, that is right continuous and with $\mathcal{F}_{0}$ containing all the subsets having measure zero. We consider a one-dimensional Wiener process $W(t)$ on this filtered probability space.

Consider the $k$-dimensional stochastic differential equation, for some multi-dimensional Wiener process $B(t)$ :

$$
d x(t)=f(x(t), t) d t+g(x(t), t) d B(t), \quad \text { with } x(0)=x_{0} .
$$

A solution is denoted by $x\left(t, x_{0}\right)$. Assume that $f(0, t)=g(0, t)=0$ for all $t \geq 0$, so that the origin point is an equilibrium point of equation (2.1).

By $\mathcal{L}$ we denote the infinitesimal generator (see for instance [12]) associated with the function displayed in equation (2.1), defined for a function $V(t, x) \in C^{1,2}\left(\mathbb{R}_{+} \times \mathbb{R}^{k}\right)$.

Definition 2.2 (See [13]) The equilibrium $x=0$ of the system (2.1) is said to be almost surely exponentially stable if for each initial value $x_{0}$ in a given subset, we have

$$
\limsup _{t \rightarrow \infty} \frac{1}{t} \ln \left|x\left(t, x_{0}\right)\right|<0 \quad \text { (a.s.). }
$$

The limit $\lim \sup _{t \rightarrow \infty} \frac{1}{t} \ln x(t)$ is called the Lyapunov exponent of $x(\cdot)$.

The following lemma was utilized in [9] and proved in [14]. For completeness we include the simple proof.

Lemma 2.3 For $k \in \mathbb{N}$, let $X(t)=\left(X_{1}(t), X_{2}(t), \ldots, X_{k}(t)\right)$ be a bounded $\mathbb{R}^{k}$-valued function and let $\left(t_{0, n}\right)$ be any increasing unbounded sequence of positive real numbers. Then there is a family of sequences $\left(t_{l, n}\right)$ such that, for each $l \in\{1,2, \ldots, k\},\left(t_{l, n}\right)$ is a subsequence of $\left(t_{l-1, n}\right)$ and the sequence $X_{l}\left(t_{l, n}\right)$ converges to a chosen limit point of the sequence $X_{l}\left(t_{l-1, n}\right)$. 
Proof Let $b$ be an upper bound for the functions $X_{i}(t)$. In the compact set $[0, b]$, we can choose a limit point in the closure of the set $\left\{X_{1}\left(t_{0, n}\right) \mid n \in \mathbb{N}\right\}$ and select a convergent subsequence $\left(t_{1, n}\right)$ of $\left(t_{0, n}\right)$ for which the limit is the chosen limit point. In the same way we can start with the sequence $\left(t_{1, n}\right)$ and pick a subsequence $\left(t_{2, n}\right)$ for which $\left(X_{2}\left(t_{2, n}\right)\right)$ is convergent, etc.

The function that we now introduce will be important in the stability analysis. Consider any $p \in[0,1]$ and let $q=1-p$. Now we define the function

$$
h: \mathbb{R}_{++} \rightarrow \mathbb{R}_{+} \quad \text { by the rule } x \mapsto \frac{1}{x}(p(1-x)+q x)^{2} .
$$

Let $h_{*}:[0,1] \rightarrow \mathbb{R}_{+}$be the function defined by

$$
h_{*}(p)= \begin{cases}4 p(1-2 p) & \text { for } 0 \leq p \leq \frac{1}{3} \\ (1-p)^{2} & \text { for } \frac{1}{3}<p \leq 1\end{cases}
$$

Proposition 2.4 Let $p$, $h$ and $h_{*}$ be as above, and let $h_{1}=\left.h\right|_{(0,1]}$ be the (domain-) restriction of $h$ to $(0,1]$. Then $h_{*}(p)$ is the absolute minimum of $h_{1}$.

Proof If $p=\frac{1}{2}$, then $h(x)=(4 x)^{-1}$ and the result follows easily. Thus, for the remainder of the proof we exclude the case $p=\frac{1}{2}$. Then we observe that $h$ tends to $+\infty$ if $x \rightarrow 0^{+}$and also, $h$ tends to $+\infty$ as $x \rightarrow+\infty$. Using calculus we find that $h^{\prime}(x)$ is continuous on $\mathbb{R}_{++}$ and has exactly one root $x_{0}$, which is $x_{0}=p \cdot|q-p|^{-1}$. Therefore, the minimum of $h_{1}$ is $h\left(x_{0}\right)$ whenever $x_{0} \leq 1$ and is $h(1)$ otherwise. Further, $x_{0} \leq 1$ if and only if $0 \leq p \leq \frac{1}{3}$. The rest of the proof follows readily.

\section{The model}

Melesse and Gumel [11] present a model for a disease of SEIRS type that may cause different stages of infectiousness in a patient. In a special case of the mentioned model, in this paper we study the effect of stochastic perturbations on the stability of the disease-free equilibrium. The population, which at any time $t$ consists of $N(t)$ individuals, is regarded as being divided into four compartments or classes. These are called the susceptible, exposed, infectious and removed classes. Their sizes, at any time $t$, are denoted by $S(t), E(t)$, $I(t)$ and $R(t)$, respectively. The equations of motion of the system are assumed to be given by the system (3.1) of stochastic differential equations. If $\sigma=0$ then the system reduces to a system of ode, which can be called the underlying deterministic model or the underlying system of ode. For the system (3.1), the underlying system of ode coincides with a special case of the model in [11]. Inflow into the population is assumed to be all into the class of susceptibles, and it is at a rate $\mu_{0} K$. Additionally there is flow from the recovered class into the class of susceptibles at a rate $\alpha_{3} R$, due to loss of infection-acquired immunity. The mortality rates in the different classes are denoted by $\mu_{i}(i=0,1,2,3)$ and this allows for higher mortality rates in classes which have been affected by the disease, such as also in [15] of Beretta et al. Hence the condition (3.2) below. The symbol $\beta$ denotes the effective contact rate. The parameters $\alpha_{1}$ and $\alpha_{2}$ determine the rates at which individuals in the population pass from classes $E$ to $I$ and (respectively) from $I$ to $R$. 
We further assume that $W$ is a standard Brownian motion. The aim of the paper is to have stochastic perturbations on the transmission rate. We do this by introducing two complementary pairs of stochastic perturbation terms. The non-negative constants $\sigma, p$ and $q$ are such that $\sigma$ determines the intensity of the perturbation, while $p$ and $q$ are the relative weights attached to the split parts of the perturbation. We assume that

$$
0 \leq p \leq 1 \quad \text { and } \quad p+q=1 \quad(\text { see Remark 3.3(b)) }
$$

The first pair of perturbation terms $(-\sigma p S E d W$ and $+\sigma p S E d W)$ constitutes randomness in the transmission rate from the class $S$ to the class $E$. Let us explain the presence of the factor $E$ (instead of $I$ ) in this component of the perturbation. We note that, for any equilibrium point $P^{*}$ of the underlying system of ode, there is a proportionality,

$$
I^{*}=\frac{\alpha_{1}}{\alpha_{2}+\mu_{2}} E^{*},
$$

and this motivates the presence of the factor $E$ in the first pair of complementary perturbation terms. This form of the first pair of terms is particularly significant since we are specifically concerned with what happens near disease-free equilibrium. The second pair of complementary perturbation terms can be understood in view of the infection ultimately driving the susceptibles (via the $E$ class) into the $I$ class. The shorter the average latent period, the more relevant does the latter perturbation become. All the parameters are non-negative or positive constants. So for instance, if $\alpha_{3}=0$, then the model is said to be of the SEIR type, but for $\alpha_{3} \neq 0$, the model is referred to as SEIRS.

The system of stochastic differential equations is as follows:

$$
\begin{aligned}
& d S=\left[\mu_{0}(K-S)-\beta S I+\alpha_{3} R\right] d t-\sigma S(p E+q I) d W, \\
& d E=\left[\beta S I-\left(\alpha_{1}+\mu_{1}\right) E\right] d t+\sigma p S E d W, \\
& d I=\left[\alpha_{1} E-\left(\alpha_{2}+\mu_{2}\right) I\right] d t+\sigma q S I d W, \\
& d R=\left[\alpha_{2} I-\left(\alpha_{3}+\mu_{3}\right) R\right] d t .
\end{aligned}
$$

Throughout the paper we assume that

$$
\mu_{0} \leq \min \left\{\mu_{1}, \mu_{2}, \mu_{3}\right\}
$$

The basic reproduction number of the underlying deterministic model, see [11], is

$$
\mathcal{R}=\frac{\alpha_{1} \beta K}{\left(\mu_{1}+\alpha_{1}\right)\left(\mu_{2}+\alpha_{2}\right)} .
$$

The following invariant $\mathcal{R}_{\sigma}$ of the model (3.1) shall feature in the main theorem on almost sure extinction of the $I$-class. In describing $\mathcal{R}_{\sigma}$ we use the number $h_{*}=h_{*}(p)$ from Section 2:

$$
\mathcal{R}_{\sigma}=\frac{\alpha_{1} \beta K}{\left(\mu_{1}+\alpha_{1}\right)\left(\mu_{2}+\alpha_{2}+\frac{1}{2} \sigma^{2} K^{2} h_{*}\right)} .
$$


We introduce the following set:

$$
\Delta_{K}=\left\{x \in \mathbb{R}_{++}^{4} \mid x_{1}+x_{2}+x_{3}+x_{4} \leq K\right\}
$$

Proposition 3.1 Suppose that, for some T, there is a local solution

$$
X(t)=(S(t), E(t), I(t), R(t)) \quad \text { on } t \in[0, T)
$$

for the system, with $X(t) \in \mathbb{R}_{+}^{4}$ for each $t \in[0, T)$. If $N(0) \leq K$, then $N(t) \leq K$ for each $t \in[0, T)$.

Proof Given any such local solution $X(t)$, then

$$
\frac{d(N-K)}{d t}=-\mu_{0}(N-K)-\left(\mu_{1}-\mu_{0}\right) E-\left(\mu_{2}-\mu_{0}\right) I-\left(\mu_{3}-\mu_{0}\right) R .
$$

The condition $X(t) \in \mathbb{R}_{++}^{4}$ together with $\mu_{0} \leq \min \left\{\mu_{1}, \mu_{2}, \mu_{3}\right\}$ ensures that

$$
\left(\mu_{1}-\mu_{0}\right) E+\left(\mu_{2}-\mu_{0}\right) I+\left(\mu_{3}-\mu_{0}\right) R \geq 0 .
$$

Consequently,

$$
\frac{d(N-K)}{d t}+\mu_{0}(N-K)=-\left(\mu_{1}-\mu_{0}\right) E-\left(\mu_{2}-\mu_{0}\right) I-\left(\mu_{3}-\mu_{0}\right) R \leq 0 .
$$

Solution of the first order linear ordinary differential equation reveals that if $N(0)<K$, then $N(t)<K$ for all $t \in[0, T)$.

We now prove the existence of solutions which are almost surely global and positive.

Theorem 3.2 Given any initial value $X_{0}=\left(S_{0}, E_{0}, I_{0}, R_{0}\right) \in \Delta_{K}$, then the system (3.1) admits a unique solution $X(t)=(S(t), E(t), I(t), R(t))$ on $t \geq 0$, and this solution remains in $\Delta_{K}$ almost surely.

Proof The coefficients of the system (3.1) are locally Lipschitz continuous. By [13], Theorem 3.5, for the given initial value $X_{0} \in \Delta_{K}$ there is a unique local solution $X(t)$ over the interval $t \in\left[0, \tau_{\text {en }}\right)$, where $\tau_{\text {en }}$ is the explosion time.

There is a number $m_{0} \in \mathbb{N}$ which is sufficiently large to allow $S_{0}, E_{0}, I_{0}, R_{0} \in\left(1 / m_{0}, K\right)$. For each $n \in \mathbb{N} \cap\left[m_{0}, \infty\right)$, let us write

$$
D_{n}=\left\{t \in\left[0, \tau_{\mathrm{en}}\right): S(t) \leq \frac{1}{n} \text { or } E(t) \leq \frac{1}{n} \text { or } I(t) \leq \frac{1}{n} \text { or } R(t) \leq \frac{1}{n}\right\} .
$$

Then we define stopping times $\tau_{n}$ and $\tau_{\infty}$ by taking $\tau_{n}$ to be the infimum of $D_{n}$ if $D_{n} \neq \emptyset$ and otherwise $\tau_{n}=\infty$. The set $D_{\infty}$ and the random variable $\tau_{\infty}$ are defined as

$$
\begin{aligned}
& D_{\infty}=\left\{t \in\left[0, \tau_{\mathrm{en}}\right): S(t) \leq 0 \text { or } E(t) \leq 0 \text { or } I(t) \leq 0 \text { or } R(t) \leq 0\right\} \\
& \tau_{\infty}=\lim _{n \rightarrow \infty} \tau_{n}=\inf D_{\infty} .
\end{aligned}
$$


For each $\gamma>0$, let $\Omega_{(\gamma)}$ be the subset of $\Omega$ defined thus:

$$
\Omega_{(\gamma)}=\left\{\in \Omega \mid \tau_{\infty}(w) \leq \gamma\right\} .
$$

We shall prove by contradiction that $\tau_{\text {en }}=\infty$ (a.s.). So let us assume to the contrary that there exist $T, C \in \mathbb{R}$ with $C>0$, and with $T<\tau_{\text {en }}$ such that $\mathbb{P}\left(\Omega_{(T)}\right)=C$.

Let us define the function $V_{0}(X)$, for $X=(S, E, I, R)$, by the formula:

$$
V_{0}(X)=\ln \frac{K}{S}+\ln \frac{K}{E}+\ln \frac{K}{I}+\ln \frac{K}{R}
$$

By Proposition 3.1, each of the four terms of $V_{0}(X(t))$ are non-negative for every $t \in\left[0, \tau_{\infty}\right)$. We set up a contradiction by calculating upper and lower bounds on expectations of $V_{0}$. Firstly we calculate an upper bound. For every $u \in\left[0, \tau_{\infty} \wedge T\right)$ we have

$$
\begin{aligned}
d V_{0}(X(u))= & \frac{-1}{S(u)}\left\{\left[\mu_{0}(K-S(u))-\beta S(u) I(u)+\alpha_{3} R(u)\right] d u\right. \\
& -\sigma S(u)(p E(u)+q I(u)) d W(u)\} \\
& -\frac{1}{E(u)}\left\{\left[\beta S(u) I(u)-\left(\alpha_{1}+\mu_{1}\right) E(u)\right] d u+\sigma p S(u) E(u) d W(u)\right\} \\
& -\frac{1}{I(u)}\left\{\left[\alpha_{1} E(u)-\left(\alpha_{2}+\mu_{2}\right) I(u)\right] d u+\sigma q S(t) I(u) d W(u)\right\} \\
& -\frac{1}{R(u)}\left\{\alpha_{2} I(u)-\left(\alpha_{3}+\mu_{3}\right) R(u)\right\} d u \\
& +\frac{1}{2}\left\{[\sigma(p E(u)+q I(u))]^{2}+(\sigma p S(u))^{2}+(\sigma q S(u))^{2}\right\} d u .
\end{aligned}
$$

We remove some negative terms and deduce the following inequality:

$$
\begin{aligned}
d V_{0}(X(u)) \leq & {[\beta I(u)] d u+\sigma(p E(u)+q I(u)) d W(u)+\left(\alpha_{1}+\mu_{1}\right) d u } \\
& -\sigma p S(u) d W(u)+\left(\alpha_{2}+\mu_{2}\right) d u-\sigma q S(u) d W(u)+\left(\alpha_{3}+\mu_{3}\right) d u \\
& +\frac{1}{2}\left\{(\sigma(p E(u)+q I(u)))^{2}+(\sigma p S(u))^{2}+(\sigma q S(u))^{2}\right\} d u .
\end{aligned}
$$

Now let

$$
\rho=\beta K+\left(\alpha_{1}+\mu_{1}\right)+\left(\alpha_{2}+\mu_{2}\right)+\left(\alpha_{3}+\mu_{3}\right)+\frac{1}{2}(\sigma(p+q) K)^{2}+\frac{1}{2}(\sigma p K)^{2}+\frac{1}{2}(\sigma q K)^{2},
$$

and for $t \in\left[0, \tau_{\infty} \wedge T\right]$, let $M_{0}(t)$ be

$$
M_{0}(t)=\sigma \int_{0}^{t}[p E(t)+q I(u)-p S(u)-q S(u)] d W(u) .
$$

Now we have the following inequality:

$$
\int_{0}^{t} d V_{0}(X(u)) \leq \rho t+M_{0}(t)
$$


Therefore, for any $k \in \mathbb{N} \cap\left[m_{0}, \infty\right)$ we have

$$
V_{0}\left(X\left(t \wedge \tau_{k}\right)\right)-V_{0}(X(0)) \leq \rho\left(t \wedge \tau_{k}\right)+M_{0}\left(t \wedge \tau_{k}\right) \quad(\text { a.s. })
$$

The stochastic process $M_{0}(t)$ is a local martingale and therefore for any $m \in \mathbb{N} \cap\left[m_{0}, \infty\right)$ we have $\mathbb{E}\left[M_{0}\left(t \wedge \tau_{m}\right)\right]=M_{0}(0)=0$. Consequently,

$$
\mathbb{E}\left[V_{0}\left(X\left(T \wedge \tau_{m}\right)\right)\right] \leq \rho\left(T \wedge \tau_{m}\right)+V_{0}(X(0)) \leq \rho T+V_{0}(X(0))
$$

and we have the upper bound which we set out to find. We now search for a lower bound for $\mathbb{E}\left[V_{0}\left(X\left(T \wedge \tau_{m}\right)\right)\right]$. Note that if $w \in \Omega_{(T)}$ and we evaluate $V_{0}(X(\zeta))$ for $\zeta=w\left(\tau_{m}\right)$, then we get

$$
V_{0}(X(\zeta)) \geq \ln (m K)
$$

We can deduce the lower bound:

$$
\mathbb{E}\left[V_{0}\left(X\left(T \wedge \tau_{m}\right)\right)\right] \geq C \ln (m K)
$$

These two bounds yield

$$
C \ln (m K) \leq \mathbb{E}\left[V_{0}\left(X\left(T \wedge \tau_{m}\right)\right)\right] \leq \rho T+V_{0}(X(0))
$$

We can choose a value of $m$ sufficiently big, so that

$$
C \ln (m K)>\rho T+V_{0}(X(0))
$$

leading to a contradiction. Therefore we must have $\tau_{\infty}=\infty$ almost surely. This completes the proof of Theorem 3.2.

Remark 3.3 (a) In the remainder of this paper we assume that sample paths are restricted to $\Omega_{0}$, which is defined as follows:

$$
\Omega_{0}=\left\{\omega \in \Omega \mid(S(t, \omega), E(t, \omega), I(t, \omega), R(t, \omega)) \in \Delta_{K} \text { for all } t \geq 0\right\} .
$$

(b) Let us briefly consider a slightly different form of the stochastic perturbation. In the first equation of the model (3.1), the $(d S)$ equation, let us consider a perturbation of the form $\left(\sigma_{1} E+\sigma_{2} I\right) S d W$, with $\sigma_{1}$ and $\sigma_{2}$ both non-negative and at least one of them being non-zero. Now let $\sigma=\sigma_{1}+\sigma_{2}$. We set $p=\sigma_{1} / \sigma$ and $q=\sigma_{2} / \sigma$. Then $p, q \in[0,1], p+q=1$ and

$$
\left(\sigma_{1} E+\sigma_{2} I\right) S d W=\sigma S(p E+q I) d W
$$

The introduction of $p$ and $q$ simplifies the analysis when we get to deal with the function $h(\cdot)$. 


\section{Stability theorems}

The concept of stability of a deterministic system of differential equations ramifies into different forms when dealing with stochastic differential equations. In this paper we shall focus on almost sure exponential stability, which is conceptually uncomplicated. We prove that when the basic reproduction number $\mathcal{R}$ of the underlying deterministic model is below unity, then the disease-free equilibrium is almost surely exponentially stable. We also prove stronger results on $I(t)$ converging to zero, in terms of the analog $\mathcal{R}_{\sigma}$ of $\mathcal{R}$.

Theorem 4.1 If $\mathcal{R}<1$, then the disease-free equilibrium of the system (3.1) is almost surely exponentially stable.

The proof of Theorem 4.1 will be presented following a discussion which is relevant to all the stability results that we derive in this paper.

Item 4.2 A construction and notation.

The following construction is crucial for the proofs of the stability theorems. We fix a positive real number $b$ and let $a \geq 0$ and $a_{1} \geq 0$. Let us write $(S(t), E(t), I(t), R(t))=X(t)$. We define the following stochastic processes:

$$
\begin{aligned}
& z(X(t))=a(K-S(t))+b E(t)+I(t)+a_{1} R(t) \\
& Q(t)=\frac{K-S(t)}{z(X(t))}, \quad E_{z}(t)=\frac{E(t)}{z(X(t))}, \quad I_{z}(t)=\frac{I(t)}{z(X(t))} \quad \text { and } \quad R_{z}(t)=\frac{R(t)}{z(X(t)} .
\end{aligned}
$$

Note that for every $t>0$ we have

$$
a Q(t)+b E_{z}(t)+I_{z}(t)+a_{1} R_{z}(t)=1
$$

Since (see Remark 3.3) we assume the sample paths to be in the subset $\Omega_{0}$, it follows that $z(X(t))>0$ for all $t>0$. Let

$$
V(X(t))=\ln z(X(t))
$$

For every sample path $w$ of the Wiener process $W(t)$, there exists an unbounded increasing sequence $\left(\tau_{n}^{w}\right)$ of positive time values for which

$$
\limsup _{t \rightarrow \infty} \mathcal{L} V(X(t, w))=\lim _{n \rightarrow \infty} \mathcal{L} V\left(X\left(\tau_{n}^{w}, w\right)\right) .
$$

Fix such a sequence. Then by Lemma 2.3 there exists a subsequence $\left(t_{n}^{w}\right)$ for which the following limits exist:

$$
\begin{aligned}
& q=\lim _{n \rightarrow \infty} Q\left(X\left(t_{n}^{w}, w\right)\right), \quad f=\lim _{n \rightarrow \infty} E_{z}\left(X\left(t_{n}^{w}, w\right)\right), \quad i=\lim _{n \rightarrow \infty} I_{z}\left(X\left(t_{n}^{w}, w\right)\right), \\
& r=\lim _{n \rightarrow \infty} R_{z}\left(X\left(t_{n}^{w}, w\right)\right) \quad \text { and } \quad s=\lim _{n \rightarrow \infty} S\left(t_{n}^{w}, w\right) .
\end{aligned}
$$

Let us write

$$
\Lambda(w)=\lim _{n \rightarrow \infty} \mathcal{L}\left(V\left(X\left(t_{n}^{w}, w\right)\right)\right) .
$$


Item 4.3 A useful inequality for $\mathcal{L} V(X(u))$.

Using Itô's formula we can express the stochastic process $V(X(t))$ as

$$
V(X(t))=V(X(0))+\int_{0}^{t} \mathcal{L} V(X(u)) d u+M_{1}(t)
$$

with $M_{1}(t)$ being the Itô integral

$$
M_{1}(t)=\int_{0}^{t}(\sigma a) \frac{p S(u) E(u)+q S(u) I(u)}{z(X(u))}+(\sigma b) \frac{p S(u) E(u)}{z(X(u))}+(\sigma) \frac{q S(u) I(u)}{z(X(u))} d W(u) .
$$

Since

$$
\begin{aligned}
& (\sigma a) \frac{p S(u) E(u)+q S(u) I(u)}{z(X(u))}+(\sigma b) \frac{p S(u) E(u)}{z(X(u))}+(\sigma) \frac{q S(u) I(u)}{z(X(u))} \\
& \leq \sigma a\left(p K \frac{1}{b}+q K\right)+\sigma b p K \frac{1}{b}+\sigma q K
\end{aligned}
$$

and the latter is a (bounded) fixed number, it follows that

$$
\lim _{t \rightarrow \infty} \frac{1}{t} \int_{0}^{t}\left[(\sigma a) \frac{p S(u) E(u)+q S(u) I(u)}{z(X(u))}+(\sigma b) \frac{p S(u) E(u)}{z(X(u))}+(\sigma) \frac{q S(u) I(u)}{z(X(u))}\right]^{2} d u<\infty
$$

Thus we may apply the strong law of large numbers for local martingales, as from [16] for instance, and we deduce that

$$
\lim _{t \rightarrow \infty} \frac{1}{t} M_{1}(t)=0 \quad \text { (a.s.). }
$$

Since also

$$
\lim _{t \rightarrow \infty} \frac{1}{t} V(0)=0
$$

it follows that

$$
\limsup _{t \rightarrow \infty} \frac{1}{t} V(X(t))=\limsup _{t \rightarrow \infty} \frac{1}{t} \int_{0}^{t} \mathcal{L} V(X(u)) d u \quad \text { (a.s.). }
$$

Now we calculate $\mathcal{L} V(X(t))$.

$$
\begin{aligned}
\mathcal{L} V(X)= & \frac{1}{z}\left[-a\left(\mu_{0}(K-S)-\beta S I+\alpha_{3} R\right)+b\left(\beta S I-\left(\alpha_{1}+\mu_{1}\right) E\right)\right. \\
& \left.+\left(\alpha_{1} E-\left(\alpha_{2}+\mu_{2}\right) I\right)+a_{1}\left(\alpha_{2} I-\left(\alpha_{3}+\mu_{3}\right) R\right)\right] \\
& -\frac{(\sigma S)^{2}}{2 z^{2}}\left(a^{2}(p E+q I)^{2}+2 a b(p E+q I) p E+2 a(p E+q I) q I\right) \\
& -\frac{(\sigma S)^{2}}{2 z^{2}}\left((p b E)^{2}+2 p q b E I+(q I)^{2}\right) .
\end{aligned}
$$


For our further analysis it will suffice to have a suitable function dominating $\mathcal{L} V$. From the last equation we obtain

$$
\begin{aligned}
\mathcal{L} V(X) \leq & \frac{1}{z}\left[-a\left(\mu_{0}(K-S)-\beta S I\right)+b\left(\beta S I-\left(\alpha_{1}+\mu_{1}\right) E\right)+\left(\alpha_{1} E-\left(\alpha_{2}+\mu_{2}\right) I\right)\right. \\
& \left.+a_{1}\left(\alpha_{2} I-\left(\alpha_{3}+\mu_{3}\right) R\right)\right]-\frac{(\sigma S)^{2}}{2 z^{2}}\left((p b E)^{2}+2 p q b E I+(q I)^{2}\right) .
\end{aligned}
$$

Now we introduce notation from Item 4.2, to obtain the following inequality:

$$
\begin{aligned}
\mathcal{L} V(X) \leq & -a\left(\mu_{0} Q-\beta S I_{z}\right)+b\left(\beta S I_{z}-\left(\alpha_{1}+\mu_{1}\right) E_{z}\right)+\left(\alpha_{1} E_{z}-\left(\alpha_{2}+\mu_{2}\right) I_{z}\right) \\
& +a_{1}\left(\alpha_{2} I_{z}-\left(\alpha_{3}+\mu_{3}\right) R_{z}\right)-\frac{(\sigma S)^{2}}{2}\left(p b E_{z}+q I_{z}\right)^{2} .
\end{aligned}
$$

Remark 4.4 In the proofs of the stability theorems we shall need to prove that, for paths $w \in \Omega_{0}$,

$$
\limsup _{t \rightarrow \infty} \frac{1}{t} \int_{0}^{t} \mathcal{L} V(X(u, w)) d u<0
$$

To this end we note here that it suffices to prove that

$$
\limsup _{t \rightarrow \infty} \mathcal{L} V(X(t, w))<0
$$

Proof of Theorem 4.1 Since $\mathcal{R}<1$ we have the inequality

$$
c \beta K-\left(\alpha_{2}+\mu_{2}\right)<0, \quad \text { with } c=\frac{\alpha_{1}}{\mu_{1}+\alpha_{1}} .
$$

Now we can choose $a>0$ sufficiently small such that

$$
(c+a) \beta K-\left(\alpha_{2}+\mu_{2}\right)<0 .
$$

We can also choose positive real numbers $a_{0}$ and $a_{1}$ sufficiently small such that

$$
\left(c+a+\frac{a_{0}}{\alpha_{1}+\mu_{1}}\right) \beta K-\left(\alpha_{2}+\mu_{2}\right)+a_{1} \alpha_{2}<0 .
$$

Now let $b=c+a_{0}\left(\mu_{1}+\alpha_{1}\right)^{-1}$, and with these values of $a, b$ and $a_{1}$ we define $z(X(t))$ as in Item 4.2. It suffices to prove that $\Lambda<0$. We modify the inequality (4.4) ignoring the last term, using $S<K$ and noting that $b\left(\alpha_{1}+\mu_{1}\right)-\alpha_{1}=a_{0}$. Then we deduce the following inequality:

$$
\mathcal{L} V(X) \leq-a \mu_{0} Q+(a+b) \beta K I_{z}-a_{0} E_{z}-\left(\alpha_{2}+\mu_{2}\right) I_{z}+a_{1} \alpha_{2} I_{z}-a_{1}\left(\alpha_{3}+\mu_{3}\right) R_{z} .
$$

We form limits as in equations (4.2a) and (4.2b), and after rearranging the terms we obtain

$$
\Lambda \leq-a \mu_{0} q+\left[(a+b) \beta K-\left(\alpha_{2}+\mu_{2}\right)+a_{1} \alpha_{2}\right] i-a_{0} f-a_{1}\left(\alpha_{3}+\mu_{3}\right) r .
$$


The coefficients of $q, f$ and $r$ are negative, and by inequality (4.5) the coefficient of $i$ is also negative. Since $a q+b f+i+a_{1} r=1$, it follows that at least one of these limits $\{q, f, i, r\}$ must be non-zero. Hence $\Lambda<0$ and the proof is complete.

Theorem 4.1 implies that if a disease-free equilibrium is locally asymptotically stable with respect to the underlying ode-system, then it is almost surely exponentially stable with respect to the stochastic model, in particular, the perturbations do not disrupt the stability of the disease-free equilibrium.

Remark 4.5 In the sequel we shall use a special form of the inequality (4.4), taking $a=$ $a_{1}=0$. Then $z=b E+I$ and from (4.4) we obtain the inequality

$$
\mathcal{L} V(X) \leq b\left(\beta S I_{z}-\left(\alpha_{1}+\mu_{1}\right) E_{z}\right)+\left(\alpha_{1} E_{z}-\left(\alpha_{2}+\mu_{2}\right) I_{z}\right)-\frac{(\sigma S)^{2}}{2}\left(p b E_{z}+q I_{z}\right)^{2} .
$$

Since $b E_{z}=1-I_{z}$, and with $h(\cdot)$ being the function as in equation (2.2), we can write

$$
\left(p b E_{z}+q I_{z}\right)^{2}=I_{z} h\left(I_{z}\right)
$$

Since $0<I_{z}<1$, by Proposition 2.4 we have $h\left(I_{z}\right) \geq h_{*}(p)$. Therefore we can write

$$
\mathcal{L} V(X) \leq b\left(\beta S I_{z}-\left(\alpha_{1}+\mu_{1}\right) E_{z}\right)+\left(\alpha_{1} E_{z}-\left(\alpha_{2}+\mu_{2}\right) I_{z}\right)-\frac{(\sigma S)^{2}}{2} I_{z} h_{*}(p)
$$

We now present the main result of this paper, which proves that the stochastic perturbation improves the stability of the disease-free equilibrium for small values of the perturbation parameter.

Theorem 4.6 If the following conditions hold:

(1) $\mathcal{R}_{\sigma}<1$,

(2) $\sigma^{2} \leq \frac{c \beta}{K h_{*}}$ with $c=\frac{\alpha_{1}}{\mu_{1}+\alpha_{1}}$,

then $(E(t), I(t))$ almost surely converges exponentially to 0 .

Proof Let us assume the conditions (1) and (2) of the theorem to hold. In particular then, the condition (1) is equivalent to

$$
c \beta K-\left(\alpha_{2}+\mu_{2}\right)-\frac{1}{2} \sigma^{2} K^{2} h_{*}<0 .
$$

Choose any positive number $a_{3}$ to be sufficiently small such that

$$
\left(c+\frac{a_{3}}{\alpha_{1}+\mu_{1}}\right) \beta K-\left(\alpha_{2}+\mu_{2}\right)-\frac{1}{2} \sigma^{2} K^{2} h_{*}<0 .
$$

This can be written

$$
b \beta K-\left(\alpha_{2}+\mu_{2}\right)-\frac{1}{2} \sigma^{2} K^{2} h_{*}<0, \quad \text { with } b=c+\frac{a_{3}}{\alpha_{1}+\mu_{1}} .
$$


Using this value of $b$ in the inequality (4.7) while $a=0=a_{1}$, yields

$$
\mathcal{L} V(X) \leq I_{z} g(S)-a_{3} E_{z}, \quad \text { where } g(S)=b \beta S-\left(\alpha_{2}+\mu_{2}\right)-\frac{(\sigma S)^{2}}{2} h_{*} .
$$

The quadratic function $g(\zeta)$ of equation (4.9) reaches an absolute maximum when $\zeta=$ $b \beta\left(\sigma^{2} h_{*}\right)^{-1}$. By assumption (2) it follows that $c \beta\left(\sigma^{2} h_{*}\right)^{-1} \geq K$. Therefore also

$$
\frac{b \beta}{\sigma^{2} h_{*}}>\frac{c \beta}{\sigma^{2} h_{*}} \geq K
$$

Considering that $0<S \leq K$, it follows that $g(S) \leq g(K)$ and therefore

$$
\mathcal{L} V(X) \leq I_{z} g(K)-a_{3} E_{z}
$$

Therefore (see the notation in Item 4.2) we have

$$
\Lambda \leq i g(K)-a_{3} f
$$

Now we observe that $g(K)$ coincides with the left hand side of the inequality (4.8). Since at least one of $f$ and $i$ must be non-zero, it follows that $\Lambda<0$. This completes the proof of Theorem 4.6.

In Section 5 we shall further reflect on Theorem 4.6. Also, Theorem 4.8 below combines very well with Theorem 4.6. However, while our main result Theorem 4.6 focused on small perturbations, let us briefly address the case of larger perturbations. The following theorem asserts that, for sufficiently large values of the perturbation parameter $\sigma$, the disease will eventually vanish from the population.

Theorem 4.7 The pair $(E(t), I(t))$ almost surely converges exponentially to 0 if

$$
\sigma^{4}>\frac{(c \beta)^{2}}{\left(\alpha_{2}+\mu_{2}\right) h_{*}^{2}} \quad \text { with } c=\frac{\alpha_{1}}{\mu_{1}+\alpha_{1}} .
$$

Proof Let $a_{4}$ be sufficiently small to support the inequality:

$$
\sigma^{4}>\frac{\left[\left(c+\frac{a_{4}}{\mu_{1}+\alpha_{1}}\right) \beta\right]^{2}}{\left(\alpha_{2}+\mu_{2}\right) h_{*}^{2}}
$$

and let $b=c+a_{4}\left(\alpha_{1}+\mu_{1}\right)^{-1}$. Now we revisit the construction presented under Item 4.2, using the constant $b$ as selected and $a=0=a_{1}$. Then similar to the proof of Theorem 4.6, we obtain an inequality,

$$
\mathcal{L} V(X) \leq I_{z} g(S)-a_{4} E_{z}, \quad \text { where } g(S)=b \beta S-\left(\alpha_{2}+\mu_{2}\right)-\frac{(\sigma S)^{2}}{2} h_{*} .
$$

The inequality $\sigma^{4}>\frac{(b \beta)^{2}}{\left(\alpha_{2}+\mu_{2}\right) h_{*}^{2}}$ is equivalent to

$$
\left(\frac{b \beta}{\sigma^{2} h_{*}}\right)^{2}-\left(\alpha_{2}+\mu_{2}\right)<0
$$


Therefore,

$$
\begin{aligned}
g(S) & =-\frac{\sigma^{2} h_{*}}{2}\left(S^{2}-\frac{2 b \beta}{\sigma^{2} h_{*}} S\right)-\left(\alpha_{2}+\mu_{2}\right) \\
& =-\frac{\sigma^{2} h_{*}}{2}\left(S-\frac{b \beta}{\sigma^{2} h_{*}}\right)^{2}+\left(\frac{b \beta}{\sigma^{2} h_{*}}\right)^{2}-\left(\alpha_{2}+\mu_{2}\right) \\
& <-\frac{\sigma^{2} h_{*}}{2}\left(S-\frac{b \beta}{\sigma^{2} h_{*}}\right)^{2} \\
& <0 .
\end{aligned}
$$

This implies that $\Lambda \leq i g(s)-a_{4} f<0$, and the proof is complete.

Theorem 4.8 If $(E(t), I(t))$ almost surely converges exponentially to 0 , then

$$
\lim _{t \rightarrow \infty} S(t)=K \quad \text { (a.s.) } \quad \text { and } \quad \lim _{t \rightarrow \infty} R(t)=0 \quad \text { (a.s.). }
$$

Proof This proof is by contradiction, so let us suppose, to the contrary, that (on a subset $\Omega_{\lim }$ of $\Omega$ of positive measure) we have $\lim _{t \rightarrow \infty}(K-S(t))+R(t)>0$. Let $z$ be as in Item 4.2, with $a=b=a_{1}=1$. Then, since $\lim _{t \rightarrow \infty} E(t)=0$ while $\lim _{t \rightarrow \infty}(K-S(t))+R(t)>0$, it follows that $f=0$ on $\Omega_{\lim }$. Similarly it follows that $i=0$ on $\Omega_{\lim }$. Therefore from the inequality (4.4) it follows that on $\Omega_{\text {lim }}$ we have

$$
\Lambda \leq-\mu_{0} q-\left(\alpha_{3}+\mu_{3}\right) r
$$

Therefore, $\Lambda<0$. This implies that $z$ converges to 0 and, consequently, that $\lim _{t \rightarrow \infty}(K-$ $S(t))+R(t)=0$, which is a contradiction. This completes the proof.

Remark 4.9 (a) Theorem 4.6 is much more significant than Theorem 4.7 because in disease modeling, in practice one is more interested in smaller perturbations rather than the larger perturbations. Let us denote the bounds on $\sigma$ specified in Theorems 4.6 and 4.7 by $\theta_{1}$ and $\theta_{2}$ respectively. If $\theta_{1}>\theta_{2}$, then these theorems can be combined, guaranteeing the disease-free equilibrium to be almost surely exponentially stable irrespective of the magnitude of $\sigma$.

(b) Of course, Theorem 4.8 serves to extend Theorems 4.6 and 4.7 .

\section{Simulations}

Theorem 4.6 suggests that $\mathcal{R}_{\sigma}$ is an approximation for a threshold that decides stability in a way similar to the basic reproduction number. Simulations show that it is a rather useful approximation. For a non-negative stochastic process, almost sure convergence to 0 can be tested by computing the (approximation over finitely many paths, of the) mean of sample paths. If the mean of $I$ is not asymptotically stable, then $I$ is not almost surely exponentially stable. The simulations that were run produced trajectories of the mean of $I$ which consistently appears to converge to a value which is smaller than, or at least not bigger than, in the deterministic case.

These simulations are obtained by considering an influenza infection of the type in [11] and [17]. The relevant parameter values for $\alpha_{1}, \alpha_{3}, \mu_{0}, \mu_{1}$ and $\mu_{3}$ are taken directly from [11] and other parameters values are derived. Our value for $1 / \alpha_{2}$ is obtained by taking the 
sum of the average times spent in the $I_{(\cdot)}$-compartments (of [11]). The value of $\mu_{2}$ is taken as $1.025 \mu_{0}$. The parameter $\beta$ is not kept fixed in these simulations. Here we note that infections that are aerially transmitted will spread faster when people are in high density locations with poor ventilation. So for instance the same disease has a higher value for the effective contact rate, when considered in a concentration camp as compared to an ordinary small village or rural area.

Initial values in millions are $S(0)=6, E(0)=2, I(0)=0.9$ and $R(0)=1.05$.

Using the Euler-Maruyama scheme, we simulate the trajectories of one sample path of the $I$-class of the model,

the mean of $I$, taken over 1,000 sample paths and indicated as 'I (1000ave)', and

the I-class trajectory 'I determ' (broken line) of the underlying deterministic model, with the parameter values as explained above. We take

$K=10$ (in millions),

and the values of the other parameters are all given, with one-day as time unit, in the accompanying Table 1.

Parameters such as $p$ and $\sigma$ are difficult to compute. We choose $p=0.333$ for simulation. The parameters $\beta$ and $\sigma$ are varied in order to obtain different values of $\mathcal{R}_{\sigma}$.

In Figure 1 we show trajectories for the case $\beta=0.0235$ and $\sigma=0.05$, for which we get $\mathcal{R}_{\sigma}=1.1429$. In this case we cannot deduce stability of the disease-free equilibrium from Theorem 4.6, since condition (1) is not satisfied. In fact we observe the mean value of $I$ as seeming to converge to a positive value. The given $I$-path also does not seem to converge to 0 . We do note, however, that after 400 days (a relatively long period) it gives a mean $I$ value (computed as 6,600), which is lower than in the deterministic case $(19,400)$.

In Figure 2 we show a case in which we take $\beta=0.021, \sigma=0.08$, and we calculate $\mathcal{R}=1.0497$ and $\mathcal{R}_{\sigma}=0.9800 \leq 1$. However, condition (2) is not satisfied. The $I$-trajectory shown does not seem to converge to 0 , although the mean seems to converge to 0 . This further demonstrates the need for condition (2) in Theorem 4.6 (other than condition (2) just being utilized in the proof). In this case the deterministic model has a non-trivial equilibrium value $I^{*}$ for $I$. For the stochastic case we observe that the mean seems to converge to a value smaller than $I^{*}$.

Table 1 Numerical values of the fixed parameters

\begin{tabular}{ll}
\hline Parameter & Value \\
\hline$\mu_{0}$ & $(60 \times 365)^{-1}=4.566 \times 10^{-5}$ \\
$\mu_{1}$ & $4.566 \times 10^{-5}$ \\
$\mu_{2}$ & $1.025 \mu_{0}=4.680 \times 10^{-5}$ \\
$\mu_{3}$ & $4.566 \times 10^{-5}$ \\
$\alpha_{1}$ & $1.9^{-1}=0.5263$ \\
$\alpha_{2}$ & 0.2 \\
$\alpha_{3}$ & $(83.33)^{-1}=0.012$ \\
\hline
\end{tabular}

Figure 1 Condition (1) of Theorem 4.6 is violated.

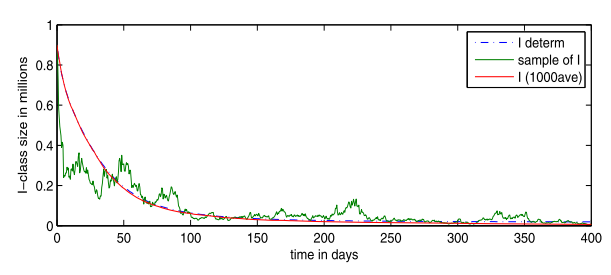


Figure 2 Condition (2) of Theorem 4.6 is violated.

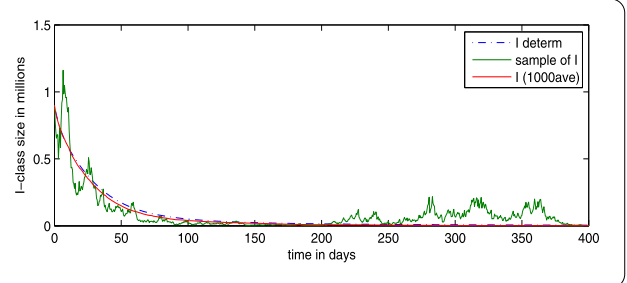

Figure 3 Theorem 4.6 guarantees the almost sure extinction of the $I$-class.

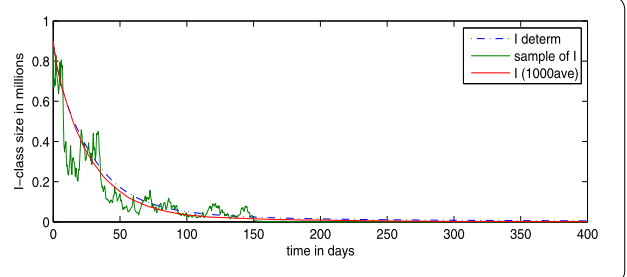

In Figure 3 we use $\beta=0.0215, \sigma=0.05$, and then we have $\mathcal{R}=1.0247$ and $\mathcal{R}_{\sigma}=0.99700$. This time the parameters selection fulfills all the conditions of Theorem 4.6, and indeed what we see appears to be in line with the assertion of the theorem.

\section{Conclusion}

In this paper we constructed an SEIRS model, with stochastic perturbations which can be viewed as linked to the transmission rate out of the class of susceptibles. We proved that the system of stochastic differential equations permits solutions that are almost surely global and positive. The model permits a disease-free equilibrium which we showed to be almost surely exponentially stable whenever the basic reproduction number of the underlying deterministic model is below unity, and even slightly beyond under given conditions.

Biologically we observe, in particular, the following effect of a stochastic perturbation on the disease transmission in the case of a deterministic compartmental model which allows for a latently infectious class. Given a small stochastic perturbation on the transmission rate from susceptible to infectious via the latent phase, the stability of the disease-free state will be improved if both components of the perturbation are non-trivial. If any one of the components of the perturbation is zero, then the stability will not be disturbed. The simulations confirm the proven results and also provide further insights, such as about the behavior of the mean of the $I$-class trajectories.

Competing interests

The author declares that he has no competing interests.

Acknowledgements

The author acknowledges financial support by the National Research Foundation of South Africa.

\section{Publisher's Note}

Springer Nature remains neutral with regard to jurisdictional claims in published maps and institutional affiliations.

Received: 9 January 2016 Accepted: 31 March 2017 Published online: 11 April 2017

References

1. Cai, H, Luo, X: Stochastic control of an epidemic process. Int. J. Syst. Sci. 254, 821-828 (1994)

2. Ishikawa, M: Optimal strategies for vaccination using the stochastic SIRV model. Trans. Inst. Syst. Control Inf. Eng. 25(12), 343-348 (2012)

3. Witbooi, PJ, Muller, GE, van Schalkwyk, GJ: Vaccination control in a stochastic SVIR epidemic model. Comput. Math. Methods Med. 2015, Article ID 271654 (2015). 
4. Yang, Q, Mao, X: Extinction and recurrence of multi-group SEIR epidemic models with stochastic perturbations. Nonlinear Anal., Real World Appl. 14(3), 1434-1456 (2013)

5. Yuan, C, Jiang, D, O'Regan, D, Agarwal, RP: Stochastically asymptotically stability of the multi-group SEIR and SIR models with random perturbation. Commun. Nonlinear Sci. Numer. Simul. 17, 2501-2516 (2012)

6. Zhao, J, Liu, M, Wang, W, Yang, P: The stability of SI epidemic model in complex networks with stochastic perturbation. Abstr. Appl. Anal. 2014, Article ID 610959 (2014)

7. Chen, G, Li, T, Liu, C: Lyapunov exponent of a stochastic SIRS model. C. R. Math. 351(1-2), 33-35 (2013)

8. Gray, A, Greenhalgh, D, Hu, L, Mao, X, Pan, J: A stochastic differential equation SIS epidemic model. SIAM J. Appl. Math. 71(3), 876-902 (2011)

9. Witbooi, PJ: Stability of an SEIR epidemic model with independent stochastic perturbations. Physica A 392(20), 4928-4936 (2013)

10. Li, MY, Muldowney, JS, van den Driessche, P: Global stability of SEIRS models in epidemiology. Can. Appl. Math. Q. 7(4), 409-425 (1999)

11. Melesse, DY, Gumel, AB: Global asymptotic properties of an SEIRS model with multiple infectious stages. J. Math. Anal. Appl. 366, 202-217 (2010)

12. Øksendal, B: Stochastic Differential Equations: An Introduction with Applications. Springer, New York (1998)

13. Mao, X: Stochastic Differential Equations and Applications. Horwood, Chichester (1997)

14. Witbooi, PJ: Stability of stochastic model of an SIR epidemic with vaccination. Acta Biotheor. (2017) doi:10.1007/s10441-017-9308-5

15. Beretta, E, Hara, T, Ma, W, Takeuchi, Y: Global asymptotic stability of an SIR epidemic model with distributed time delay. Nonlinear Anal., Theory Methods Appl. 47(6), 4107-4115 (2001)

16. Feller, W: An Introduction to Probability Theory and Its Applications, vol. II. Wiley, New York (1966)

17. Nuño, M, Reichert, TA, Chowell, G, Gumel, AB: Protecting residential care facilities from pandemic influenza. Proc. Natl. Acad. Sci. USA 105(30), 10625-10630 (2008)

\section{Submit your manuscript to a SpringerOpen ${ }^{\circ}$ journal and benefit from:}

- Convenient online submission

- Rigorous peer review

- Immediate publication on acceptance

- Open access: articles freely available online

- High visibility within the field

- Retaining the copyright to your article 